\title{
Mechanisms of Hydrogen Transport in Flexible-Wall Narrow Carbon Nanotubes
}

\author{
Bin-Hao Chen, ${ }^{1}$ Chieh Kung, ${ }^{2}$ and I-Peng $\mathrm{Chu}^{3}$ \\ ${ }^{1}$ Department of Energy Application Engineering, Far East University, No. 49, Zhonghua Road, Xinshi District, \\ Tainan City 74448, Taiwan \\ ${ }^{2}$ Department of Industrial Design, Far East University, No. 49, Zhonghua Road, Xinshi District, Tainan City 74448, Taiwan \\ ${ }^{3}$ Department of Mechanical Engineering, Far East University, No. 49, Zhonghua Road, Xinshi District, Tainan City 74448, Taiwan
}

Correspondence should be addressed to Bin-Hao Chen; binhaol7@gmail.com

Received 29 April 2014; Revised 9 July 2014; Accepted 12 August 2014

Academic Editor: Xi-Qiao Feng

Copyright (C 2015 Bin-Hao Chen et al. This is an open access article distributed under the Creative Commons Attribution License, which permits unrestricted use, distribution, and reproduction in any medium, provided the original work is properly cited.

\begin{abstract}
Understanding the interaction between hydrogen and carbon nanotubes is crucial to enhancing the performance of hydrogen storage and nanofluidic carbon-adsorbent systems. Accordingly, this study performs a series of molecular dynamics simulations to investigate the transport properties of hydrogen molecules confined within a flexible narrow carbon nanotube. The tube's diameter is $10.8 \AA$ at temperatures in the range of $100 \sim 800 \mathrm{~K}$. The particle loadings inside carbon nanotubes are ranging from $0.01 \sim 1 \mathrm{No} / \AA$. The results show that the hydrogen molecules exhibit three distinct diffusion regimes, namely, single-file, Fickian, and ballistic, depending on the value of the Knudsen number. In addition, it is shown that with the Knudsen number of less than 1, the tube-wall long wavelength acoustic phonons induced Rayleigh traveling wave prompts a longitudinal wave slip and compression-expansion of the hydrogen molecule crowds within the CNT, which leads to a significant increase in the mean square displacement of the molecules.
\end{abstract}

\section{Introduction}

A hydrogen-based energy economy, properly integrated with the use of renewable energy resources, provides the potential to satisfy the energy requirements of vehicles, buildings, portable electronic devices, and so on $[1,2]$. However, in practice, the feasibility of a hydrogen economy is constrained by the difficulties involved in designing suitable hydrogen storage and delivery systems. Previous studies have shown that hydrogen gas condenses to a high density state when confined within a narrow, single-wall nanotube (SWNT) [3]. The actual mechanisms of hydrogen diffusion within SWNTs and carbon nanotubes (CNTs) are still unclear. Many researchers [1-9] have demonstrated that the structure and properties of nanometer scale materials can be quite different from those of bulk materials due to the presence of surface effects. Accordingly, lightweight carbon adsorbent materials have become interesting for possible use in a hydrogen storage system. The exceptional physical properties of CNTs, including high thermal conductivity, strong mechanical properties, excellent electrical conductivity, and so forth, render them highly promising candidates for a range of hydrogen storage and delivery applications. Consequently, the nanofluidic adsorption properties and transport mechanisms of hydrogen molecules within SWNTs and CNTs have attracted great interest in the energy industry [4-7]. According to conventional physisorption principles, the gasadsorption performance of a porous solid is maximized when the pores are no larger than a few molecular diameters [8]. Zuo et al. had conducted a series simulation and suggested an interesting result that MWCNT can be separated into SWCNT in appropriate solvents [9]. Under these conditions, the potential fields produced at the wall overlap to produce a stronger interaction force than that observed in adsorption on a simple plane. However, the mechanisms responsible for the adsorption and transportation of hydrogen in nanoporous solids or nanopores are not easily observed using experimental methods. As a result, the use of computational methods such as molecular dynamics (MD) or Monte Carlo (MC) simulations have emerged as the method of choice for examining the nanofluidic properties of liquids and gases within nanoporous materials [10]. Several groups have 
performed numerical simulations to study the adsorption of water in CNTs [11-16], while others have investigated the diffusion of pure hydrocarbon gases and their mixtures through various SWNTs with diameters ranging from 2 $8 \mathrm{~nm}[6,17,18]$. These studies focus on self- and transport diffusion coefficients of inert gases, hydrogen, and methane in infinitely long SWNTs $[19,20]$. In general, the results show that the transport rates in nanotubes are in orders of magnitude higher than those measured experimentally in zeolites or other microporous crystalline solids. In addition, it has been shown that the dynamic flow of helium and argon atoms through SWNTs is highly dependent on the surface temperature of the nanotube wall [21]. Specifically, the flow rate of the helium and argon atoms, as quantified in terms of their self-diffusion coefficients, increased with temperature due to the greater thermal activation effect.

Previous MD simulations of the nanofluidic properties of liquids and gases generally assumed the nanoporous material to have a rigid structure. However, if the nanoporous material is not in fact rigid, the simulation results may deviate from the true values by several orders of magnitude. Several researchers had investigated the conditions under which the assumption of a rigid lattice is, or is not, reasonable [22, 23]. In general, the results showed that while the use of a rigid lattice was permissible in modeling the nanofluidic properties of a gas or liquid in an unconfined condition, a flexible lattice assumption was required when simulating the properties of a fluid within a constrained channel. Moreover, in real-world conditions, the thermal fluctuations of the atoms on a CNT wall impact the diffusive behavior of the adsorbed molecules, and must therefore be taken into account. Jakobtorweihen et al. have reported careful simulations of molecular diffusion considering nanotube flexibility [24]. The reproduction of the results of a flexible carbon nanotube in a rigid nanotube simulation was excellent. Ayappa had examined the influence of nanotube flexibility on the transport diffusion of $\mathrm{CH}_{4}$ in nanotubes [25]. The inclusion of nanotube flexibility reduces the transport diffusion relative to the rigid nanotube by roughly an order of magnitude close to zero pressure. At the pressure over 1 bar the transport diffusivities for flexible and rigid nanotubes are very similar. Interestingly, whether or not it is reasonable to assume a rigid lattice in diffusion studies is far from being understood [23]. An obvious hypothesis would be that only in the case of narrow passages is flexibility very important. For the cases of gas molecules in carbon nanotubes, or other nanoporous materials, a rigid lattice is a very reasonable assumption [24]. That is to say, if one has a material in which the flexibility cannot be ignored, such as diffusion in confined space, the mechanism will be dominated by surface effect. To prove this opinion, we present herein simulations to track the MSD behavior of hydrogen diffusion inside narrow SWCNT. The simulations show that until the carbon nanotubes are filled with hydrogen molecules up to a specific cutoff density, the molecules diffuse through the nanotubes will transit to a wave manner induced by long wavelength acoustic phonons. For comparison purpose, we conduct a rigid-lattice model to provide the evidence between flexible and rigid lattice model. Results of rigidlattice model agree with A. I. Skolulidas' results. However, the MSD curves of rigid-lattice model do not reveal the jump behavior. Rigid-lattice model simulations are shown in the Appendix [Animation A1, A2; Supporting Information].

This study performs a series of MD simulations to investigate the transport properties of hydrogen molecules confined within a narrow CNT with a diameter of $10.8 \AA(\sim 1 \mathrm{~nm})$ in temperatures ranging from $100 \sim 800 \mathrm{~K}$ and particle loadings of $0.01 \sim 1 \mathrm{No} / \AA$. To ensure the validity of the simulation results, the $\mathrm{MD}$ model assumes the tube to have a flexible wall. Hydrogen molecules are treated as spherical particles. In performing the simulations, the hydrogen molecules are assumed to have a perfectly spherical shape. In addition, the interactions between the molecule and the atoms on the CNT wall and the interactions between the carbon atoms within the CNT wall are modeled using the Lennard-Jones potential $[26,27]$. The simulations focus on the hydrogen adsorption within the SWNT instead of in the interstices or the external surface of nanotube bundles. As described above, the simulations performed in this study consider a CNT with a pore size (diameter) of $10.8 \AA$. In such a small-pore regime [28], the interactions between the pore wall and the hydrogen molecules have a significant effect on the diffusion behavior of the molecules as they migrate along the CNT. The simulations performed in this study consider Knudsen numbers in the range of $0.1<\mathrm{Kn}<10$ [29]. In this particular flow regime, the local transport mechanism depends on a number of interfacial parameters, including the strength of the liquid-solid coupling, the thermal roughness of the interface, and the commensurability of the wall and liquid densities, respectively [30]. The diffusivity of the hydrogen molecules is quantified in terms of the mean square displacement (MSD) and self-diffusion coefficient under particle loadings ranging from $0.01 \sim 1 \mathrm{No} / \AA$. In diffusion studies, it is a common practice to integrate the equations of motion for hydrogen molecules confined within infinitely long nanotubes. The selfdiffusion coefficient is obtained from the average MSD of the hydrogen molecules as a function of time. General Fickiantype diffusion is described by the seminal relation

$$
\left\langle X^{2}\right\rangle=2 D t^{\gamma} \quad \text { with } \gamma=1
$$

However, for more complicated random driving forces, the diffusive behavior may be slower ( $\gamma<1$, subdiffusion) or faster ( $\gamma>1$, superdiffusion) than normal diffusion [29]. In the present simulations, the results obtained for the mean free path are used to compute the Knudsen number, and the diffusion regime is inferred by comparing the time-based variation of the MSD profile with the predicted time-dependent MSD profiles for single-file, Fickian and ballistic diffusion, respectively. In addition to analyzing the change in the diffusion regime over time, the simulations also find out an unexpected "jump phenomenon" on MSD curves. Simulations are conducted to investigate the "slip" of the hydrogen molecules caused by long wavelength acoustic phonons. Tube wall peristaltic movement activates a compression-relaxation diffusion mechanism at very low values of the Knudsen number. 

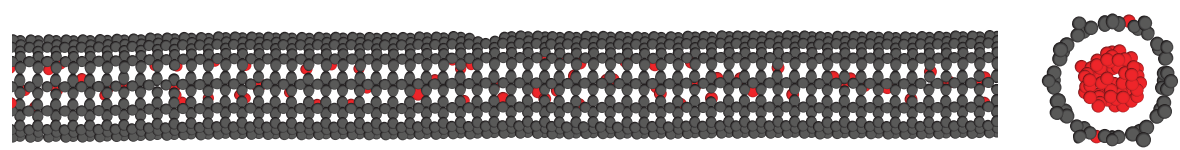

FIGURE 1: Simulation model of hydrogen molecules within SWCNT.

\section{Simulation Model}

In the simulations, the hydrogen molecules are initially dispersed with a random distribution within the CNT. Figure 1 presents a snapshot of the simulation model for the case in which the CNT contains 70 hydrogen molecules. The current simulations are performed using the methodology presented in [24], which closely mimics the actual interfacial interactions between the hydrogen molecules and the tube wall. In the simulations, the hydrogen molecules are assumed to be "free" (i.e., they can migrate freely from their initial positions) and are heated to an equilibrium state $\left(\sim 10^{4} \mathrm{fs}\right)$ via their interaction with the heated CNT wall. The CNT temperature is maintained at the specified value using the Nosé-Hoover thermostat method. In performing the simulations, two different tube-diffuser interactions take place. The interactions can be divided into two types: (1) the carbon atoms vibrate as a result of the applied temperature and collide with the hydrogen molecules near the wall, thereby imparting a transfer of thermal and mechanical energy from the wall to the hydrogen molecules, and (2) the tube is flexible; long wavelength acoustic phonons will be induced and therefore apply a peristaltic driving force to the hydrogen molecules. Since the nanotube is flexible, the lattice vibrational modes are critical. Thus, flexibility can change the morphology of nanotube following the mode of nature vibration. We use a nanotube of length $\sim 30 \mathrm{~nm}$ to sample the vibration modes accurately and avoid the size effects.

In order to accurately model the interaction between the tube wall and the hydrogen molecules, the simulations consider the case where just one hydrogen molecule exists within the tube such that the effects of inter-diffusant collisions can be ignored. As a result, the diffusion of the hydrogen molecules through the CNT is consistent with the thermal transpiration phenomenon identified by Reynolds [30-34], in which the molecules have more impact on the CNT wall than on one another. In the simulations, the diffusion of the hydrogen molecules is governed by two transient processes. In the first process, the hydrogen molecules are activated by the heated tube surface toward a thermal equilibrium state and start to diffuse within the CNT, while in the second process, the activated molecules interact with the stagnant molecules and cause these molecules to diffuse also. Thus, the moving behavior tends to become a specified diffusion type.

In Figure 1 we show a right view which is parallel with and a left view perpendicular to the pore axis. Note that carbon atoms are shown in black while hydrogen molecules are shown in red. Pore diameter is $10.8 \AA$.
TABLE 1: Parameters for the carbon interaction potential used in the simulation.

\begin{tabular}{ll}
\hline$K_{\mathrm{Cr}}=478.9 \mathrm{~kJ} \mathrm{~mol}^{-1} \AA^{-2}$ & $r_{\mathrm{C}}=1.418 \AA$ \\
$K_{\mathrm{C} \theta}=562.2 \mathrm{~kJ} \mathrm{~mol}^{-1}$ & $\theta_{\mathrm{C}}=120.00^{\circ}$ \\
$\varepsilon_{\mathrm{cc}}=0.4396 \mathrm{~kJ} \mathrm{~mol}^{-1}$ & $\sigma_{\mathrm{cc}}=3.851 \AA$ \\
\hline
\end{tabular}

TABLE 2: Morse potential dimensionless units used in the simulation.

\begin{tabular}{lcc}
\hline Unit & & Dimensionless unit \\
\hline Length & $r(\AA)$ & $r^{*}=r / r_{0}$ \\
Time step & $d t(\mathrm{~s})$ & $d t^{*}=d t \cdot \sqrt{D /\left(m \cdot r_{0}^{2}\right)}$ \\
Temperature & $T(\mathrm{~K})$ & $T^{*}=T \cdot(k / D)$ \\
Energy & $E(\mathrm{eV})$ & $E^{*}=E / D$ \\
\hline
\end{tabular}

2.1. Potential Functions. The carbon nanotube is modeled by terms describing the Morse bond and harmonic cosine of the bonding angle as $[35,36]$

$$
U\left(r_{i j}, \theta_{i j k}\right)=K_{c r}\left(\xi_{i j}-1\right)^{2}+\frac{1}{2} K_{c \theta}\left(\cos \theta_{i j k}-\cos \theta_{c}\right)^{2},
$$

where

$$
\xi_{i j}=\exp \left[-\gamma\left(r_{i j}-r_{c}\right)\right],
$$

$\theta_{i j k}$ represents all possible bending angles, and $r_{i j}$ represents all distances between bonded atoms. $K_{c r}, K_{c \theta}$ are the force constants of stretch, bend potentials, respectively. $r_{c}, \theta_{c}$ are the corresponding reference geometry parameters. The Morse stretch and angle bending parameters were first given by Guo et al. [36]. These parameters, listed in Table 1, originally derived to describe the geometry and phonon structure of graphite and fullerene crystals. A Lennard-Jones term is added to the nanotube potential to account for the steric and van der Waals carbon-carbon interaction

$$
U\left(r_{i j}\right)=4 \varepsilon_{\mathrm{cc}}\left[\left(\frac{\sigma_{\mathrm{cc}}}{r_{i j}}\right)^{12}-\left(\frac{\sigma_{\mathrm{cc}}}{r_{i j}}\right)^{6}\right] .
$$

Excluding 1-2 and 1-3 pairs, the parameters $\varepsilon_{\mathrm{cc}}$ and $\sigma_{\mathrm{cc}}$ are taken from the so-called Universal Force Field (UFF) [31-37].

The physical model scale lying at the microscopic level necessitates introducing some dimensionless units into the simulation activity. These dimensionless parameters are presented in Table 2. 


\section{Results and Discussions}

3.1. Boundary Slip due to Compression-Expansion Process. Before discussion, a key nondimensional parameter for gas micro flows is the Knudsen number $(\mathrm{Kn})$, which is defined as the ratio of the mean free path over a characteristic geometry length. A classification of the different flow regimes is given by Schaaf and Chambre [38]. We can observe that, after thermal equilibrium regime, cases with $\mathrm{Kn}<1$ represent a sharp vertical jump simultaneously in MSD curve. Next, we investigate the physical origin of the interaction behavior observed at the moment when "slip" phenomena happened.

To clarify the effect of the initial hydrogen density on the transport properties within the CNT, simulations were performed with various numbers of hydrogen molecules in the range of $0.01 \sim 1 \mathrm{No} / \AA$. In each case, the simulation results were used to compute the MSD curves over the duration of the simulation as a function of the system temperature. Figures 2(a) 2(d) present the MSD curves for temperatures of $100 \mathrm{~K}, 300 \mathrm{~K}, 500 \mathrm{~K}$, and $800 \mathrm{~K}$, respectively. For clarity, each figure shows the MSD curves only for loadings of 10 , 30, 50, 70, and 90 molecules, respectively. (Note, however, that these curves are representative of the curves obtained for all values of the particle loading.) In each figure, it can be seen that the MSD curves exhibit an increase of five to seven orders of magnitude at periodic intervals in the simulation procedure. The jumps in the MSD curves correspond to a "slip" of the hydrogen molecules along the pore axis. Previous studies have concluded that the exceptionally high transport rates observed in nanotubes are the result of the inherent smoothness of the nanotube wall surface $[39,40]$. However, the present simulation results suggest that the "slip flow" phenomenon is caused not only by the virtually frictionless interface at the CNT wall, but also by the effects of the compression-relaxation process with $\mathrm{Kn}<1$. The results presented in Figure 2 indicate that following the slip events, the hydrogen molecules diffuse in a ballistic mode until the end of the simulation. Previous studies have reported that boundary slips at the nanoscale occur in both the positive and the negative directions [41-43]. The present simulation results suggest that the occurrence of positive and negative slip in gas flows within a confined space may be a result of a compression-expansion effect.

3.2. Mechanisms of Hydrogen Diffusion. The hydrogen diffusion mechanism within the CNT can be inferred from the scaling behavior of the MSD curves over time. The present simulation results indicate that hydrogen diffusion occurs after $10^{4}$ picoseconds and tends toward an anomalous diffusion mode in either the subdiffusion regime $(\gamma<1)$ or the superdiffusion regime $(\gamma>1)$ as the simulation time approaches $50 \mathrm{~ns}$. In this study, the diffusion mode of the hydrogen molecules is quantified using the following formulation:

$$
\left\langle X^{2}\right\rangle=2 C t^{\gamma}
$$

where $C$ is the diffusion mobility and $\gamma$ is a coefficient whose value depends on both the pore diameter and the particle loading. In other words, the index $\gamma$ reflects the easiness with which the molecules can (or cannot) pass one another within the CNT.

The MSD profiles presented in Figure 4 show that the hydrogen molecules experience three distinct diffusion regimes, namely, a short ballistic regime, followed by a subdiffusive regime due to a thermal equilibrium process, and finally an anomalous-diffusion regime or a return to a ballistic-diffusion regime, depending on the value of $\mathrm{Kn}$. Similar distinct diffusion regions have been observed for the diffusion of colloids and certain lightweight molecules $[24,25,44,45]$.

To better understand the hydrogen diffusion mechanism within the present CNT, the following discussions consider the extreme case in which the nanotube contains only one molecule (i.e., free-molecular flow). Under this condition, $\mathrm{Kn}$ is larger than $100(\lambda \gg d ; \sim \infty)$; that is, the mean free path is much larger than the pore size. As a result, the migration behavior of the hydrogen atom is dominated by its interaction with the pore wall. The MD results suggest that in free molecular flow condition hydrogen molecule would be exerted a resistance force by the tube wall. The MSD curves represent a quasihorizontal trend toward the end of simulation. This coordinated motion mechanism is observed in a temperature range from $100 \mathrm{~K}$ to $800 \mathrm{~K}$. Observing the MSD profiles in Figure 4, it can be seen that the curves exhibit a quasihorizontal trend toward the end of the simulations. This characteristic is particularly pronounced at a temperature lower than $300 \mathrm{~K}$. The relatively constant value of the MSD implies that the wall exerts a resistive effect which opposes the motion of the hydrogen molecule. In practice, it is thought that this resistive force originates from the thermally-induced peristaltic motion of the CNT wall, which gives rise to a nonuniform potential field. With temperatures lower than $300 \mathrm{~K}$, the hydrogen molecule has insufficient kinetic energy to overcome this "surface roughness" effect, and thus its diffusion coefficient saturates to a constant value as the simulation proceeds. The thermally-induced roughness of the CNT surface provides an interesting new insight into the stick-slip phenomena observed in many boundary lubrication problems [40].

The present results indicate that the hydrogen molecules diffuse in a single-file mode for Knudsen numbers in the range of $2<\mathrm{Kn}<4$. In such a condition, the hydrogen molecules are so rare, and the kinetic energy of each molecule becomes so low, that the molecules cannot overtake one another as they migrate along the CNT. When the temperature is increased to $800 \mathrm{~K}$, the hydrogen molecules exhibit an anomalous diffusion mode for $0.5<\gamma<1$. In this regime, the MSD curves are characterized by a greater fluctuation due to the enhanced thermal roughness of the CNT wall. The confined hydrogen molecules diffuse in accordance with a Fickian-type mechanism in the range of $1<\mathrm{Kn}<2$ since in this particular regime, the molecules are able to pass one another easily as they travel along the CNT. However, as the value of the Knudsen Number is reduced, the diffusion mechanism transits to either superanomalous diffusion $(\gamma>1)$ or ballistic diffusion $(\gamma=2)$, depending on the particular value of $\mathrm{Kn}$. For the values of $\mathrm{Kn}$ less than 1, the diffusion mechanism 


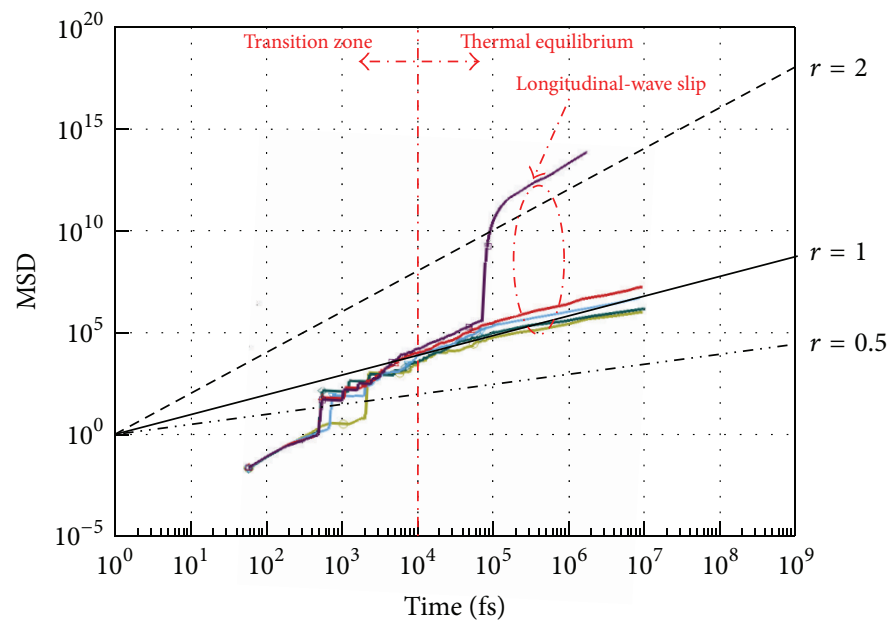

Loading density: molecules (nm)

$\begin{array}{ll}\rightarrow-1 & \triangle 7 \\ \rightarrow 3 & \square-10 \\ \rightarrow 5 & \end{array}$

(a) $100 \mathrm{~K}$

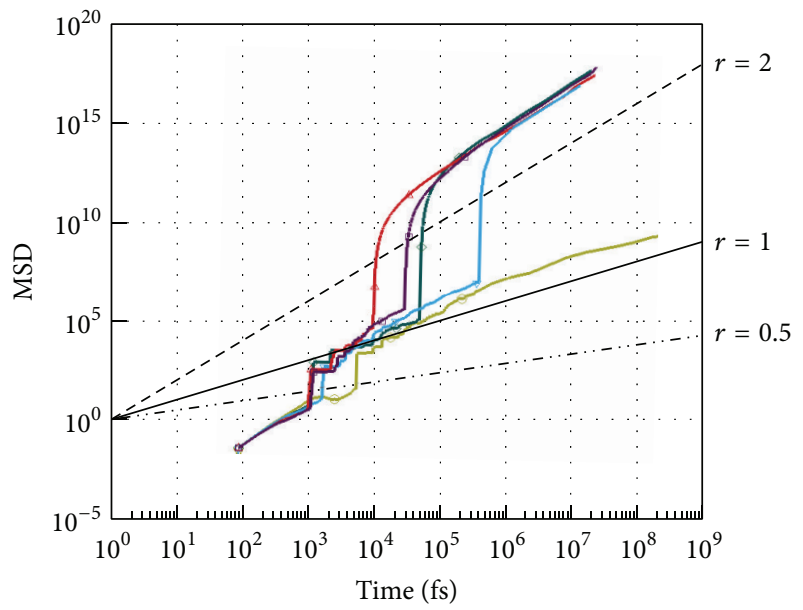

Loading density: molecules ( $\mathrm{nm})$

$$
\begin{array}{ll}
\rightarrow 1 & \rightarrow 7 \\
\rightarrow-3 & \square-10
\end{array}
$$

(c) $500 \mathrm{~K}$

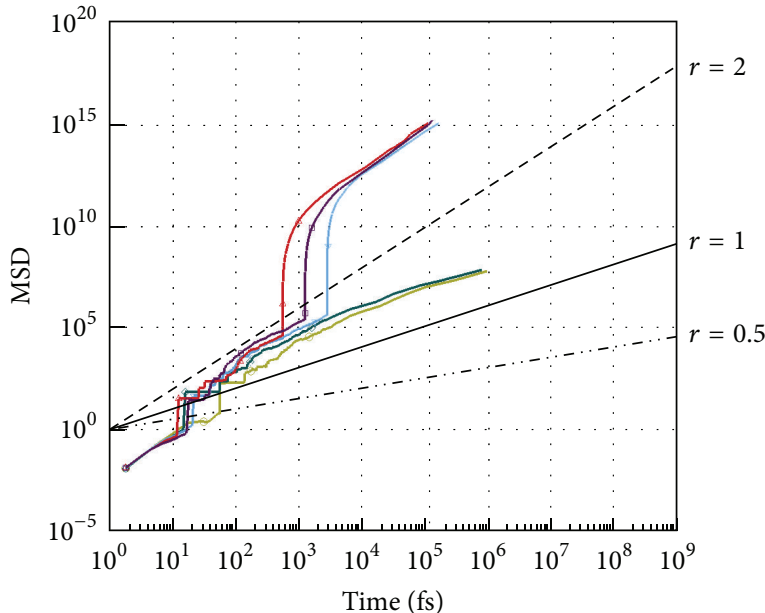

$\begin{array}{ll}\text { Loading density: molecules }(\mathrm{nm}) \\ \neg-1 & \triangle-7 \\ \rightarrow-3 & \square-10 \\ \rightarrow-5 & \end{array}$

(b) $300 \mathrm{~K}$

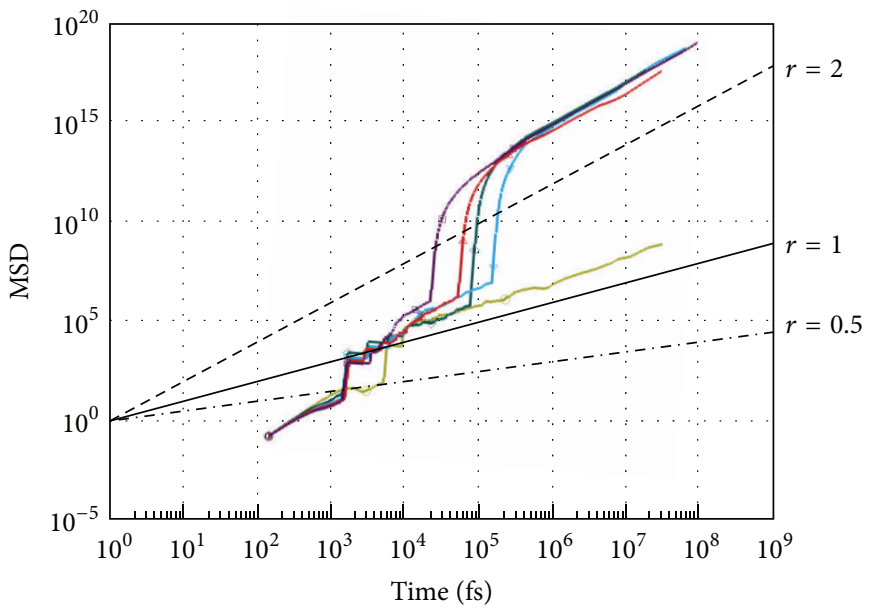

Loading density: molecules (nm)

$$
\begin{array}{ll}
\rightarrow 1 & \rightarrow 7 \\
\rightarrow 3 & \rightarrow 10 \\
\rightarrow 5 &
\end{array}
$$

(d) $800 \mathrm{~K}$

FIGURE 2: (a)-(d): mean square displacement curves, that is, mean square displacement curves of hydrogen molecules along the pore axis $\left(d z^{2} \sim \AA^{2}\right)$ as function of time for simulation temperatures of $T=100 \mathrm{~K}, 300 \mathrm{~K}, 500 \mathrm{~K}$, and $800 \mathrm{~K}$, respectively. Note that the green, turquoise, blue, red, and violet lines correspond to particle loadings of 10, 30, 50, 70, and 90 adsorbed hydrogen molecules, respectively. In addition, the three black lines represent the scaling behavior expected for the ballistic diffusion mechanism (dashed), the Fickian mechanism (solid), and the single-file diffusion mechanism (dashed-dotted), respectively.

transits to a ballistic mode as a result of the compressionexpansion process discussed in the previous section.

To understand how the transport properties of confined hydrogen molecules vary as the degree of particle loadings changes, we conducted simulations for several degree of filling ranging from 10 to $120 \mathrm{No} / \AA$ and the corresponding Knudsen numbers are located in transition flow regime. We repeat the simulation procedure up to $50 \mathrm{~ns}$ of production time. In Figure 3, we report the MSD obtained for hydrogen in partially filled SWNTs. At longer time (above $100 \mathrm{ps}$ ) the diffusion mechanism tends to become slower or faster than the Fickian-type which is dependent on Kn. In current simulation results, MSD curves indicate that after the transition zone flow mechanism becomes highly dependent on $\mathrm{Kn}$. This means that the dominating effect on the flow either will still be wall interaction or change to particle collisions. If $\mathrm{Kn}$ is greater than 1 , flow mechanism performs an anomalous type with $0.5<\gamma<1$ dominated by wall thermal 


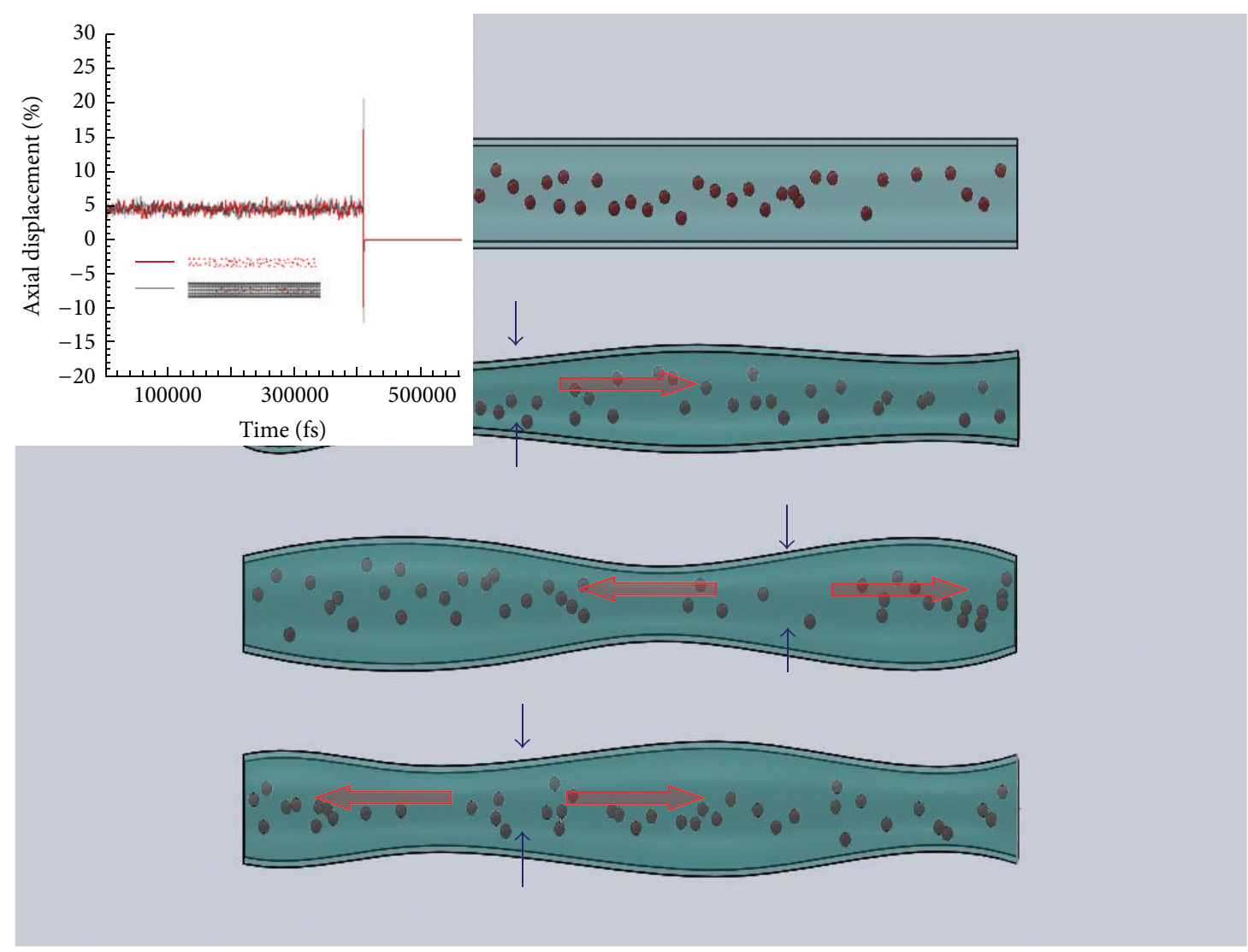

FIGURE 3: Sequence of simulation snapshots. Sequence of simulation snapshots obtained for 50 hydrogen molecules confined within SWCNT at $500 \mathrm{~K}$. The results show the formation of two neighboring molecule crowds which subsequently interact with each other via a compressionrelaxation process. Note that the inset shows the tube-wall and diffuser axial vibration displacements at $500 \mathrm{~K}$; load density $=10 \mathrm{molecules} / \mathrm{nm}$. According to the results, both the radial vibration displacements of hydrogen and tube wall present a resonance profile and the radial breathing mode is the most relevant effect in current case.

roughness. Wall fluctuation affects the hydrogen flow. When $\mathrm{Kn}$ is smaller than 1 , it is clear from visual observation of Animation 2 that particle collisions are responsible for the formation of piecewise crowd distribution of hydrogen molecules within the SWCNTs. The concerted motion of compression-relaxation transport determines the ballistictype diffusion mechanism evident from the analysis of MSD for up to 106 108 fs (see Figure 3).

3.3. Molecular Origin of Diffusion Mechanism. To understand this jump behavior by molecular origin and appreciate the molecular mechanism of hydrogen diffusion, a simulation model is proposed. Figure 3 presents a sequence of four consecutive snapshots of the simulation model for the case where the CNT contains 50 hydrogen molecules and the system temperature is equal to $500 \mathrm{~K}(\mathrm{Kn}=0.48)$. It can be seen that the hydrogen molecules have a uniform density distribution initially. However, as the thermal energy is transferred through the tube wall, the hydrogen molecules are transported in the radial direction (i.e., toward the center of the tube). The resulting collisions between the activated molecules and the stagnant molecules, together with the long wavelength acoustic phonons effect exerted by the flexible CNT wall, cause a piecewise increase in the hydrogen density along the pore axis. The piecewise molecular density distribution produces a concentration gradient across the pore and creates a driving force parallel to the pore axis. After a certain elapsed time, the peristaltic effects and the concentration gradient prompt neighboring crowds of hydrogen molecules to approach one another (i.e., to compress) in the direction indicated by the gray arrow. Interestingly, if the compression gets higher the crowd of hydrogen molecules reacts against the wall compression and moves rapidly along with the tube axis in the direction opposite to the neighbor crowd moves. As the compression effect increases, the resistive force between the hydrogen molecules within neighboring crowds also increases and eventually prompts a separation (i.e., a relaxation) of the two crowds. Therefore, a sudden increase (jump) in the MSD of the hydrogen molecules occurs. Due to the periodic boundary conditions imposed in the simulations, this compression-resistance-relaxation process is repeated periodically along the length of the CNT. And then, this piecewise density distribution performs longitudinal wave propagation. Using this model we can approach the real state of the hydrogen molecules inside SWNTs [Animation 1 (a), (b); Supporting Information]. It is worth mentioning that there exists a singular phenomenon "molecular escape through the CNT interstitial" which also 


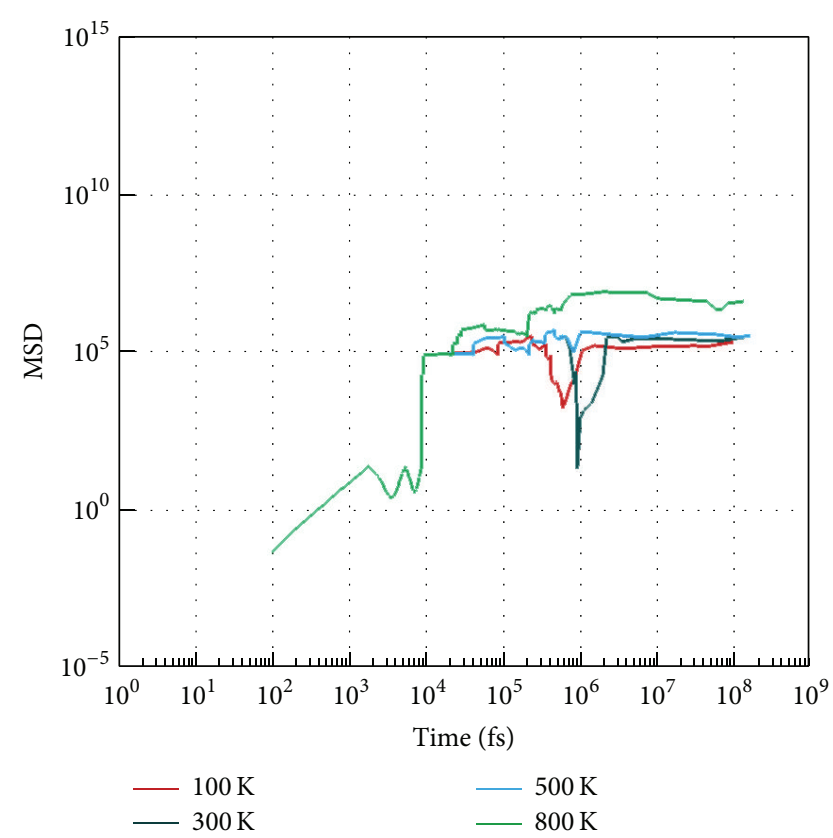

FIgURE 4: Mean square displacement associate with only one hydrogen molecule.

TABLE 3: $K n$ and $\gamma$ under different temperatures and particle loading.

\begin{tabular}{lcccccccc}
\hline$T$ & \multicolumn{2}{c}{$100 \mathrm{~K}$} & \multicolumn{2}{c}{$300 \mathrm{~K}$} & \multicolumn{2}{c}{$500 \mathrm{~K}$} & \multicolumn{2}{c}{$800 \mathrm{~K}$} \\
$K n ; \gamma$ & $K n$ & $\gamma$ & $K n$ & $\gamma$ & $K n$ & $\gamma$ & $K n$ & $\gamma$ \\
\hline Number & & & & & & & & \\
1 & $\infty$ & & $\infty$ & & $\infty$ & & $\infty$ & \\
10 & 3.75 & 0.5 & 1.575 & 1 & 2.5 & 0.85 & 1.25 & 1.16 \\
30 & 2.125 & 0.5 & 1.023 & 0.8 & 0.537 & 2 & 0.525 & 2 \\
50 & 1.0627 & 1 & 0.613 & 2 & 0.48 & 2 & 0.4625 & 2 \\
70 & 1.023 & 1 & 0.5 & 2 & 0.45 & 2 & 0.3 & 2 \\
90 & 0.43 & 2 & 0.53 & 2 & 0.48 & 2 & 0.338 & 2 \\
\hline
\end{tabular}

can release this compression pressure. This interesting behavior occurred randomly with $\mathrm{Kn}<1$ [Animation 2; Supporting Information].

\subsection{Dependence of Hydrogen Diffusion Mode on Particle} Loading. Table 3 summarizes the correlation between the value of $\mathrm{Kn}$ and the diffusion behavior of the hydrogen molecules. It can be seen that the diffusion mode of the hydrogen molecules changes from anomalous diffusion to ballistic diffusion as the value of Kn decreases.

Figure 5 shows the variation of the hydrogen selfdiffusion coefficient with the particle loading in the range of $10 \sim 120 \mathrm{No} / \AA$ as a function of the simulation temperature. The results show that the diffusion coefficient increases exponentially with an increasing particle loading. (Note that the loading is plotted in logarithmic scale.) For a loading of less than approximately 30 molecules, the diffusion coefficient is independent of the number of molecules within the CNT and is determined primarily by the effects of the interactions between the molecules and the tube wall. By contrast, for

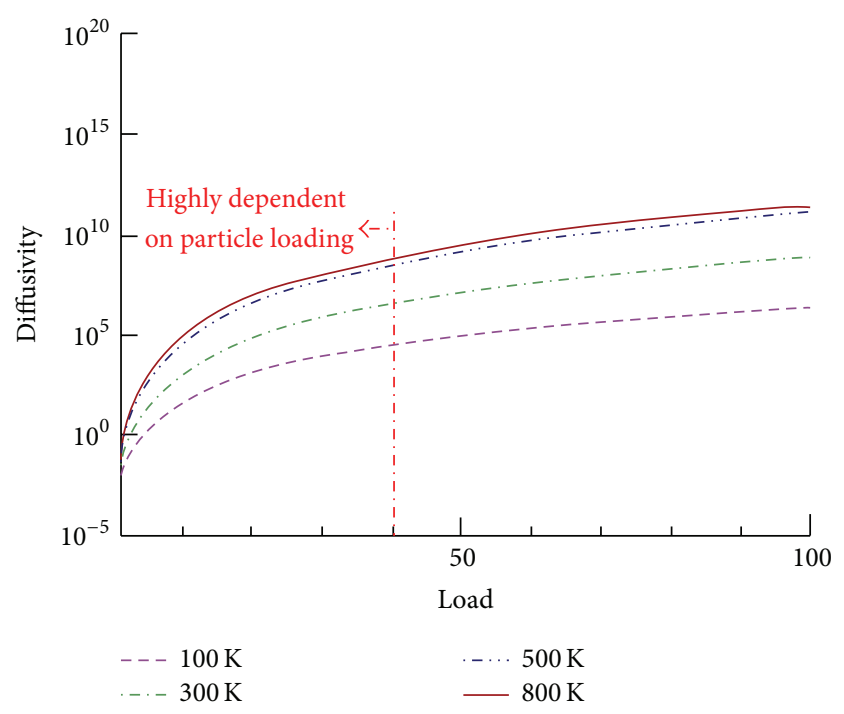

FIGURE 5: Self-diffusion coefficients. Note that the lines represent the best-fitting approximation for each set of simulation results.

loadings greater than 30 molecules, the diffusion coefficient increases rapidly as the number of molecules is increased. As the loading increases, the mean free path decreases as a result of the greater collision frequency. Consequently, at higher particle loadings, the coefficient diffusion is dominated by the interdiffusant collision frequency rather than the interaction effects of the nanotube wall. As shown in Figure 5, each curve tends to approach toward a limiting value of diffusion coefficient at temperature of $100 \mathrm{~K}, 300 \mathrm{~K}, 500 \mathrm{~K}$, and $800 \mathrm{~K}$ respectively. In Figure 5, simulation results also show that the diffusion coefficient of hydrogen inside SWCNT exists as a limiting value of $3.04 \times 10^{-3} \mathrm{~m}^{2} \mathrm{~s}^{-1}$ with $\mathrm{Kn}<1$.

\section{Conclusions}

This study has performed a series of MD simulations to investigate the diffusion behavior of hydrogen molecules within a CNT of infinite length and a diameter of $10.8 \AA$. The simulations have considered temperatures in the range 100 800 K and particle loadings ranging from 1 120 molecules/nm. The diffusion of the hydrogen molecules under each of the considered simulation conditions has been quantified by computing the variation of the mean square displacement and the self-diffusion coefficient over time. In general, the results have shown the existence of three distinct diffusion regimes, depending on the value of the Knudsen number (Kn). For values of Kn greater than 1, the hydrogen molecules exhibit an anomalous diffusion mechanism (i.e., single-file or Fickian), whereas for values of Kn less than 1, the hydrogen molecules exhibit a ballistic behavior as a result of a thermally induced compression-relaxation of the hydrogen molecule crowds within the CNT. A concerted cluster-type motion was also observed for polymers $[46,47]$ and LJ fluid $[19,48]$ in SWCNTs. However, the present simulation results differ from the results presented in these studies in that the hydrogen bonds form crowds rather than clusters due to their low 

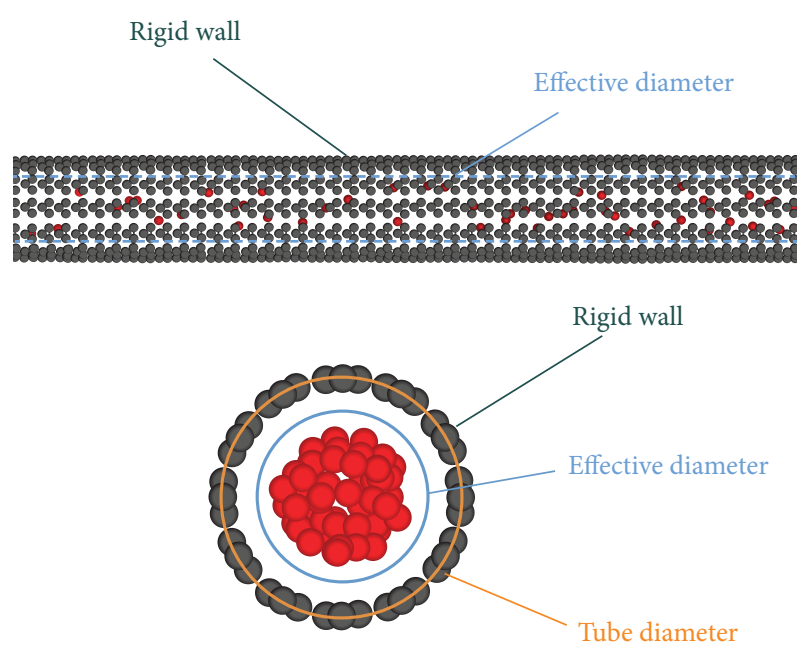

FIGURE 6: Hydrogen diffusion in rigid-lattice model (see also Animation A1, A2, in Supplementary Material available online at http://dx.doi.org/10.1155/2014/959402).

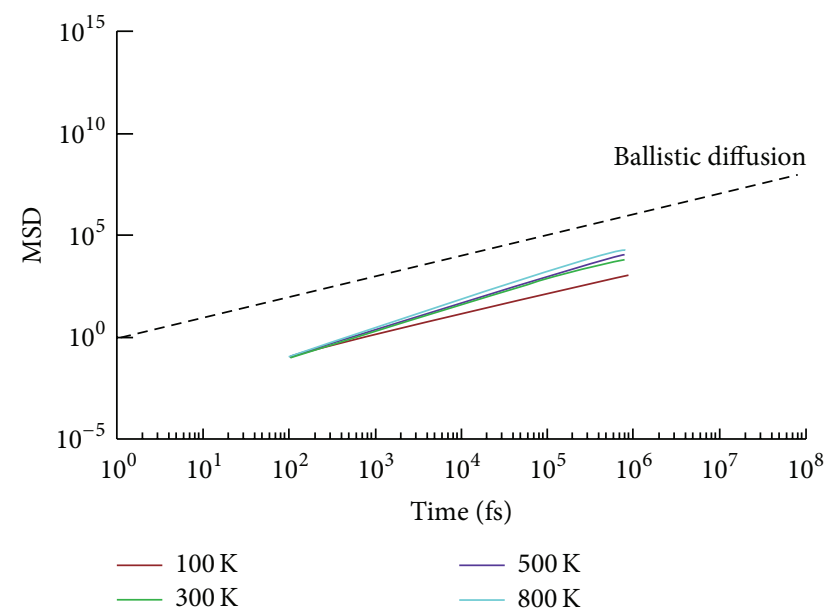

FIGURE 7: Mean square displacement curves of hydrogen molecules in the rigid-lattice SWNT as function of time for simulation temperatures of $T=100 \mathrm{~K}$ (red), $300 \mathrm{~K}$ (green), $500 \mathrm{~K}$ (blue), and $800 \mathrm{~K}$ (light blue), respectively. Note that particle loadings 70 adsorbed hydrogen molecules.

self-interaction potential. Furthermore, the ballistic motion of the hydrogen molecules prompted by the compressionrelaxation process persists for a relatively long time (i.e., $\sim 50 \mathrm{ps})$ compared to that of the clusters reported in $[19,43$, 44]. Consequently, it is inferred that simulation time in the order of tens of nanoseconds is required to reliably estimate the self-diffusion coefficients of molecules confined within a narrow CNT. Recently, Sisan and Lichter proposed that the solitons can transport water in narrow CNTs [49]. This result may give the connection between quantum sieving and hydrogen storage in adsorbents.

\section{Appendix}

To provide the evidence to support the opinion of the influences between flexible and fixed lattice model, we conduct a rigid-lattice model to simulate the hydrogen diffusion inside SWNT for comparison purpose. The nanotubes considered here are described with carbon atoms fixed during the simulations. The self-diffusion coefficients predicted for simple fluids through rigid SWCNTs can be faster than those predicted through flexible SWCNTs [35]. This effect is important at low loadings and becomes less important as the loading density increases. For the scope of the present work, which is to investigate the mechanism of hydrogen molecules diffusion within narrow SWCNTs, it is unreasonable to consider rigid SWCNTs for saving computation effort since two types of carbon atom vibrations are expected to affect the tendency of adsorbed hydrogen molecules. This study performs a series of molecular dynamics simulations to investigate the transport properties of hydrogen molecules confined within a rigid carbon nanotube with a diameter of $10.8 \AA$ at temperatures in the range of 100 800 K and high particle loadings $1 \mathrm{No} / \AA$. We never observe the MSD curves jump behavior on rigidlattice model. To assess the reliability of our results, we repeat selected simulation runs with different initial conditions. No significant difference was noticed on the conclusions here reported (see Figures 6 and 7).

\section{Conflict of Interests}

The authors declare that there is no conflict of interests regarding the publication of this paper.

\section{Acknowledgment}

The authors gratefully acknowledge the financial support provided to this study by Ministry of Science and Technology under no. 102-2221-E-269-019 -.

\section{References}

[1] L. Schlapbach and A. Züttel, "Hydrogen-storage materials for mobile applications," Nature, vol. 414, no. 6861, pp. 353-358, 2001.

[2] H.-H. Rogner, "Hydrogen technologies and the technology learning curve," International Journal of Hydrogen Energy, vol. 23, no. 9, pp. 833-840, 1998.

[3] A. C. Dillon, K. M. Jones, T. A. Bekkedahl, C. H. Kiang, D. S. Bethune, and M. J. Heben, "Storage of hydrogen in single-walled carbon nanotubes," Nature, vol. 386, no. 6623, pp. 377-379, 1997.

[4] R. M. de Vos and H. Verweij, "High-selectivity, high-flux silica membranes for gas separation," Science, vol. 279, no. 5357, pp. 1710-1711, 1998.

[5] L. N. Gergidis and D. N. Theodorou, "Molecular dynamics simulation of n-butane-methane mixtures in silicalite," Journal of Physical Chemistry B, vol. 103, no. 17, pp. 3380-3390, 1999.

[6] Z. Mao and S. B. Sinnott, "Separation of organic molecular mixtures in carbon nanotubes and bundles: molecular dynamics 
simulations," Journal of Physical Chemistry B, vol. 105, no. 29, pp. 6916-6924, 2001.

[7] K. T. Thomson and R. M. Wentzcovitch, "A density functional study of the electronic structure of sodalite," The Journal of Chemical Physics, vol. 108, no. 20, pp. 8584-8588, 1998.

[8] S. J. Gregg and K. S. W. Sing, Adsorption, Surface Area and Porosity, Academic Press, London, UK, 2nd edition, 1982.

[9] J. Zou, B. Ji, X.-Q. Feng, and H. Gao, "Self-assembly of singlewalled carbon nanotubes into multi walled carbon nanotubes in water: molecular dynamics simulations," Nano Letters, vol. 6, no. 3, pp. 430-434, 2006.

[10] I. A. Khan and K. G. Ayappa, "Density distributions of diatoms in carbon nanotubes: a grand canonical Monte Carlo study," Journal of Chemical Physics, vol. 109, no. 11, pp. 4576-4586, 1998.

[11] I. Brovchenko, A. Geiger, and A. Oleinikova, "Phase equilibria of water in cylindrical nanopores," Physical Chemistry Chemical Physics, vol. 3, no. 9, pp. 1567-1569, 2001.

[12] A. Giaya and R. W. Thompson, "Water confined in cylindrical micropores," The Journal of Chemical Physics, vol. 117, no. 7, pp. 3464-3475, 2002.

[13] W. H. Noon, K. D. Ausman, R. E. Smalley, and M. Jianpeng, "Helical ice-sheets inside carbon nanotubes in the physiological condition," Chemical Physics Letters, vol. 355, no. 5-6, pp. 445448, 2002.

[14] A. Striolo, A. A. Chialvo, K. E. Gubbins, and P. T. Cummings, "Water in carbon nanotubes: adsorption isotherms and thermodynamic properties from molecular simulation," Journal of Chemical Physics, vol. 122, no. 23, Article ID 234712, 2005.

[15] A. Striolo, K. E. Gubbins, M. S. Gruszkiewicz et al., "Effect of temperature on the adsorption of water in porous carbons," Langmuir, vol. 21, no. 21, pp. 9457-9467, 2005.

[16] A. Striolo, A. A. Chialvo, P. T. Cummings, and K. E. Gubbins, "Simulated water adsorption in chemically heterogeneous carbon nanotubes," Journal of Chemical Physics, vol. 124, no. 7, Article ID 074710, 2006.

[17] Z. Mao and S. B. Sinnott, "A computational study of molecular diffusion and dynamic flow through carbon nanotubes," Journal of Physical Chemistry B, vol. 104, no. 19, pp. 4618-4624, 2000.

[18] Z. Mao, A. Garg, and S. B. Sinnott, "Molecular dynamics simulations of the filling and decorating of carbon nanotubules," Nanotechnology, vol. 10, article 273, 1999.

[19] D. M. Ackerman, A. I. Skoulidas, D. S. Sholl, and J. K. Johnson, "Diffusivities of Ar and Ne in carbon nanotubes," Molecular Simulation, vol. 29, no. 10-11, pp. 677-684, 2003.

[20] A. I. Skoulidas, D. M. Ackerman, J. K. Johnson, and D. S. Sholl, "Rapid transport of gases in carbon nanotubes," Physical Review Letters, vol. 89, no. 18, 2002.

[21] R. E. Tuzun, D. W. Noid, B. G. Sumpter, and R. C. Merkle, "Dynamics of He/C60 flow inside carbon nanotubes," Nanotechnology, vol. 8, no. 3, pp. 112-118, 1997.

[22] T. J. H. Vlugt and M. Schenk, "Influence of framework flexibility on the adsorption properties of hydrocarbons in the zeolite silicalit," Journal of Physical Chemistry B, vol. 106, no. 49, pp. 12757-12763, 2002.

[23] P. Demontis and G. B. Suffritti, "Structure and dynamics of zeolites investigated by molecular dynamics," Chemical Reviews, vol. 97, no. 8, pp. 2845-2878, 1997.

[24] S. Jakobtorweihen, M. G. Verbeek, C. P. Lowe, F. J. Keil, and B. Smit, "Understanding the loading dependence of self-diffusion in carbon nanotubes," Physical Review Letters, vol. 95, no. 4, Article ID 044501, 2005.
[25] K. G. Ayappa, "Simulations of binary mixture adsorption in carbon nanotubes: transitions in adsorbed fluid composition," Langmuir, vol. 14, no. 4, pp. 880-890, 1998.

[26] Y. Guo, N. Karasawa, and W. A. Goddard III, "Prediction of fullerene packing in $\mathrm{C}_{60}$ and $\mathrm{C}_{70}$ crystals," Nature, vol. 351, no. 6326, pp. 464-467, 1991.

[27] W. A. Steele, The Interaction of Gas with Solid Surfaces, Pergamon Press, Oxford, UK, 1974.

[28] J. Kärger and D. M. Ruthven, Diffusion in Zeolites and Other Microporous Solids, John Wiley \& Sons, New York, NY, USA, 1992.

[29] G. Karniadakis, A. Beskok, and N. Aluru, Micro Flows: Fundamentals and Simulation, Springer, 2001.

[30] R. C. Reid, J. M. Prausnitz, and B. E. Poling, Properties of Gases and Liquids, McGraw-Hill, New York, NY, USA, 1987.

[31] O. Reynolds, "On certain dimensional properties of matter in the gaseous state. Part I. Experimental researches on thermal transpiration of gases through porous plates and on the laws of transpiration and impulsion, including an experimental proof that gas is not a continuous plenum. Part II. On an extension of the dynamical theory of gas, which includes the stresses, tangential and normal, caused by a varying condition of gas, and affords an explanation of the phenomena of transpiration and impulsion," Philosophical Transactions of the Royal Society of London, vol. 170, pp. 727-845, 1879.

[32] M. Knudsen, "Eine Revision der Gleichgewichtsbedingung der Gase. Thermische Molekular-strömung," Annals of Physics, vol. 31, pp. 205-229, 1910.

[33] M. Knudsen, "Thermischer Molekulardruck der Gase in Röhren," Annals of Physics, vol. 338, pp. 1435-1448, 1910.

[34] L. B. Loeb, "Kinetic theory of gases," Journal of the American Chemical Society, vol. 81, no. 5, pp. 1267-1267, 1959.

[35] R. E. Tuzun, D. W. Noid, B. G. Sumpter, and R. C. Merkle, "Dynamics of He/C60 flow inside carbon nanotubes," Nanotechnology, vol. 7, p. 241, 1996.

[36] Y. Guo, N. Karasawa, and W. A. Goddard III, "Prediction of fullerene packing in C60 and C70 crystals," Nature, vol. 351, no. 6326, pp. 464-467, 1991.

[37] A. K. Rappé, C. J. Casewit, K. S. Colwell, W. A. Goddard III, and W. M. Skiff, "UFF, a full periodic table force field for molecular mechanics and molecular dynamics simulations," Journal of the American Chemical Society, vol. 114, no. 25, pp. 10024-10035, 1992.

[38] S. Schaaf and P. Chambre, Flow of Rarefied Gases, Princeton University Press, Princeton, NJ, USA, 1961.

[39] M. Majumder, N. Chopra, R. Andrews, and B. J. Hinds, "Nanoscale hydrodynamics: enhanced flow in carbon nanotubes," Nature, vol. 438, no. 7064, p. 44, 2005.

[40] G. Hummer, J. C. Rasaiah, and J. P. Noworyta, "Water conduction through the hydrophobic channel of a carbon nanotube," Nature, vol. 414, no. 6860, pp. 188-190, 2001.

[41] P. A. Thompson and S. M. Troian, "A general boundary condition for liquid flow at solid surfaces," Nature, vol. 389, no. 6649, pp. 360-362, 1997.

[42] K. P. Travis, B. D. Todd, and D. J. Evans, "Departure from Navier-Stokes hydrodynamics in confined liquids," Physical Review E: Statistical Physics, Plasmas, Fluids, and Related Interdisciplinary Topics, vol. 55, no. 4, pp. 4288-4295, 1997.

[43] K. P. Travis and K. E. Gubbins, "Poiseuille flow of Lennard-Jones fluids in narrow slit pores," Journal of Chemical Physics, vol. 112, no. 4, pp. 1984-1994, 2000. 
[44] H. Ebert and K. R. Albrand, "The applicability of gas flows in the molecular range," Vacuum, vol. 13, no. 12, pp. 563-568, 1963.

[45] T. R. Cuthbert, N. J. Wagner, M. E. Paulaitis, G. Murgia, and B. D’Aguanno, "Molecular dynamics simulation of penetrant diffusion in amorphous polypropylene: diffusion mechanisms and simulation size effects," Macromolecules, vol. 32, no. 15, pp. 5017-5028, 1999.

[46] A. Striolo, "The mechanism of water diffusion in narrow carbon nanotubes," Nano Letters, vol. 6, no. 4, pp. 633-639, 2006.

[47] C. Wei and D. Strivastava, "Theory of transport of long polymer molecules through carbon nanotube channels," Physical Review Letters, vol. 91, Article ID 235901, pp. 1-4, 2003.

[48] H. Chen, J. K. Johnson, and D. S. Sholl, "Transport diffusion of gases is rapid in flexible carbon nanotubes," Journal of Physical Chemistry B, vol. 110, no. 5, pp. 1971-1975, 2006.

[49] T. B. Sisan and S. Lichter, "Solitons transport water through narrow carbon nanotubes," Physical Review Letters, vol. 112, no. 4, Article ID 044501, 2014. 

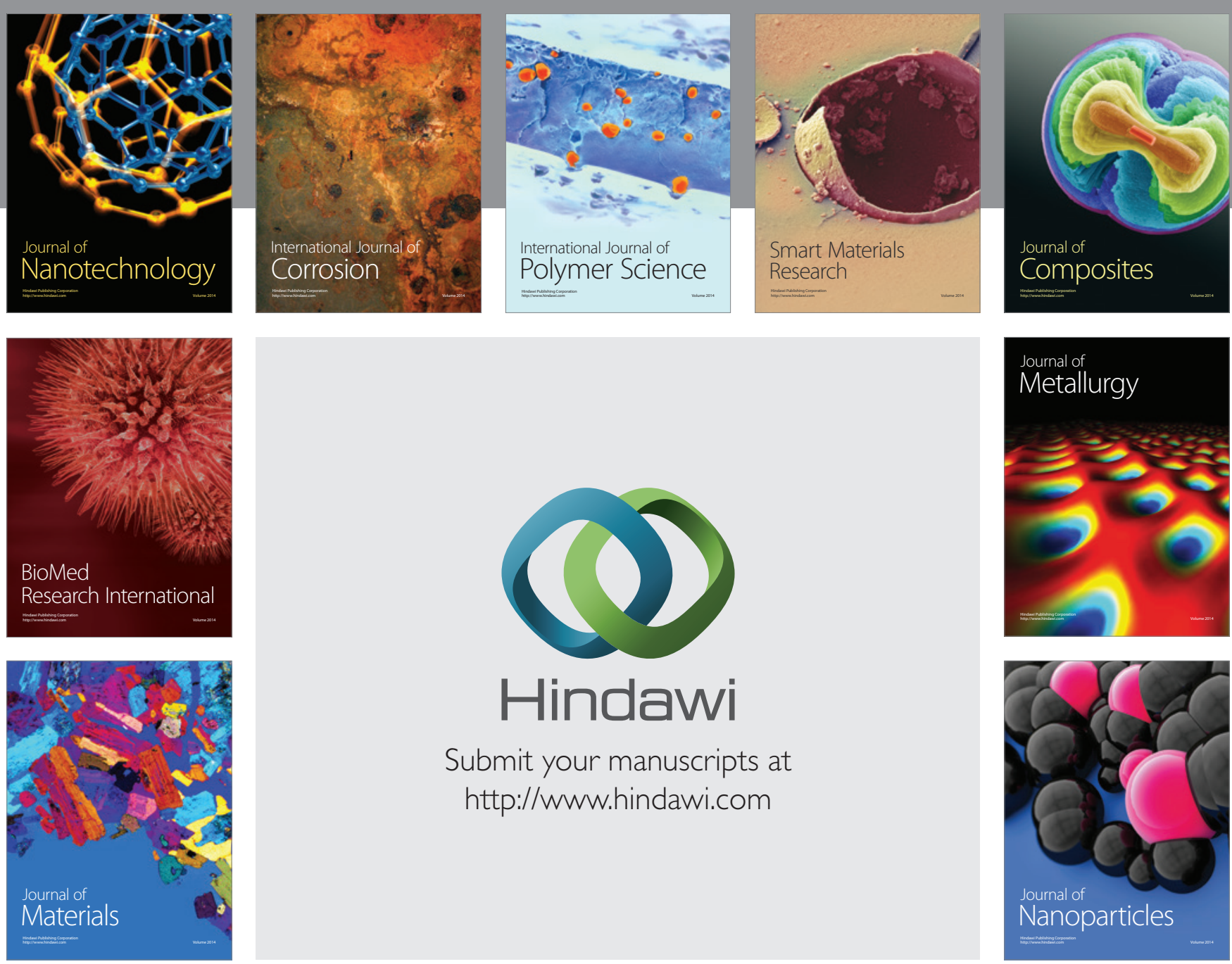

Submit your manuscripts at http://www.hindawi.com
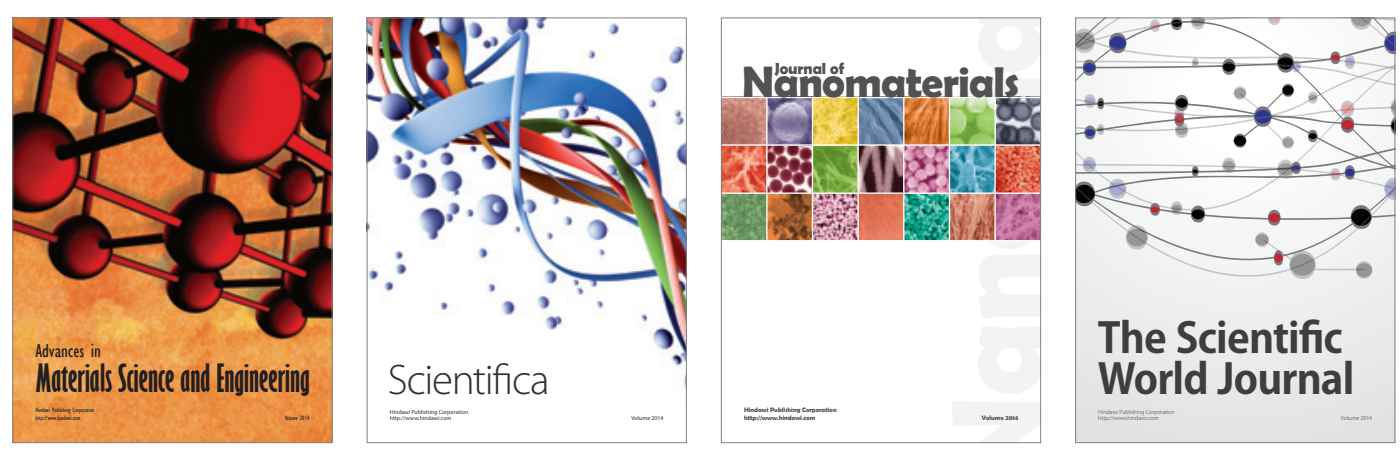

\section{The Scientific World Journal}
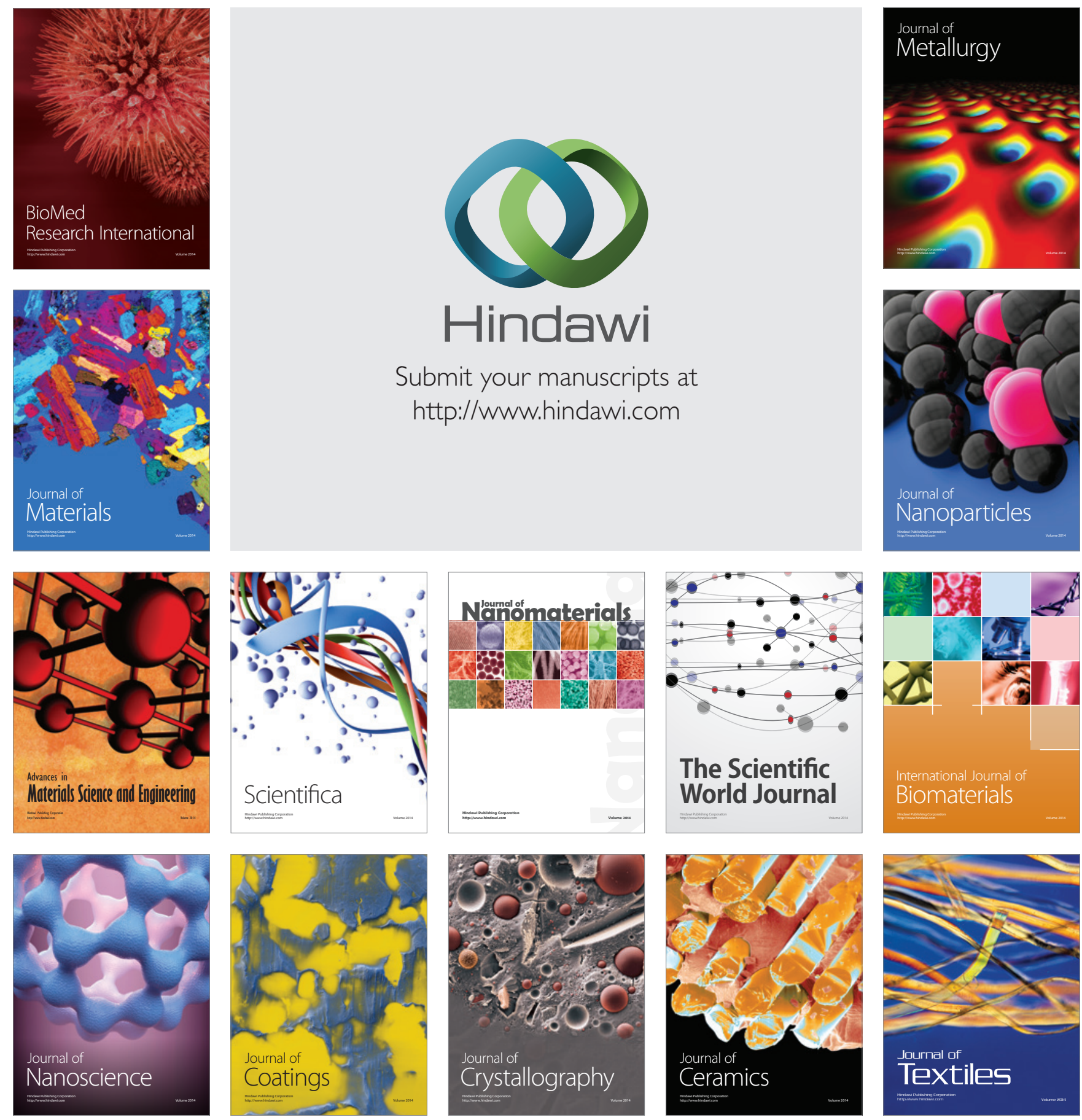